Q.-S. Chi

Nagoya Math. J.

Vol. 193 (2009), 129-154

\title{
ISOPARAMETRIC HYPERSURFACES WITH FOUR PRINCIPAL CURVATURES REVISITED
}

\author{
QUO-SHIN CHI
}

\begin{abstract}
The classification of isoparametric hypersurfaces with four principal curvatures in spheres in [2] hinges on a crucial characterization, in terms of four sets of equations of the 2nd fundamental form tensors of a focal submanifold, of an isoparametric hypersurface of the type constructed by Ferus, Karcher and Münzner. The proof of the characterization in [2] is an extremely long calculation by exterior derivatives with remarkable cancellations, which is motivated by the idea that an isoparametric hypersurface is defined by an over-determined system of partial differential equations. Therefore, exterior differentiating sufficiently many times should gather us enough information for the conclusion. In spite of its elementary nature, the magnitude of the calculation and the surprisingly pleasant cancellations make it desirable to understand the underlying geometric principles.

In this paper, we give a conceptual, and considerably shorter, proof of the characterization based on Ozeki and Takeuchi's expansion formula for the Cartan-Münzner polynomial. Along the way the geometric meaning of these four sets of equations also becomes clear.
\end{abstract}

\section{$\S 1$. Introduction}

In [2], isoparametric hypersurfaces with four principal curvatures and multiplicities $\left(m_{1}, m_{2}\right), m_{2} \geq 2 m_{1}-1$, in spheres were classified to be exactly the isoparametric hypersurfaces of FKM-type constructed by Ferus Karcher and Münzner [4]. The classification goes as follows. Let $M_{+}$be a focal submanifold of codimension $m_{1}+1$ of an isoparametric hypersurface in a sphere, and let $\mathcal{N}$ be the normal bundle of $M_{+}$in the sphere. Suppose on the unit normal bundle $U \mathcal{N}$ of $\mathcal{N}$ there hold true the four sets of equations

$$
\begin{aligned}
F_{\alpha p}^{\mu} & =F_{\alpha p-m}^{\mu}, \\
F_{a+m b}^{\alpha} & =-F_{b+m a}^{\alpha},
\end{aligned}
$$

Received April 19, 2007.

Accepted May 24, 2008.

2000 Mathematics Subject Classification: Primary 53C40.

The author was partially supported by NSF Grant No. DMS-0103838. 


$$
\begin{aligned}
F_{a+m b}^{\mu} & =-F_{b+m a}^{\mu} \\
\omega_{a}^{b}-\omega_{a+m}^{b+m} & =\sum_{p} L_{b a}^{p}\left(\theta^{p-m}+\theta^{p}\right),
\end{aligned}
$$

for some smooth functions $L_{b a}^{p}$. Here, $m:=m_{1}$ for notational ease. At $n \in U \mathcal{N}$ with base point $x$, the indices $a$ (and $b$ ), $p, \alpha, \mu$ run through, respectively, $n^{\perp}$, the subspace perpendicular to $n$ in the fiber $\mathcal{N}_{x}$, and the three eigenspaces of the shape operator $A_{n}$ with eigenvalues $0,1,-1$. Also, $F_{j a}^{i}$ is, up to constant multiples, the $(i, j)$-component of the second fundamental form in the normal $a$-direction at $x \in M_{+}$pulled back to $n \in U \mathcal{N}$, and $\theta^{i}$ and $\omega_{j}^{i}$ are the coframe and connection forms on $U \mathcal{N}$. We proved that these four sets of equations characterize an isoparametric hypersurface of FKM-type, on which the Clifford system acts on $M_{+}$.

The first three sets of equations above are algebraic whereas the last one is a system of partial differential equations. We introduced in [2] a spanning property on the 2nd fundamental form of $M_{+}$, which says that the 2 nd fundamental form is nondegenerate in the weaker sense that it is a surjective linear map from the subsapce of the direct sum of the aforementioned $\alpha$ and $\mu$ eigenspaces of the tangent space to the normal space, when one fixes any one of the two slots in the bilinear form. This spanning property turned out to be a crucial one for simplifying the four sets of equations, in that we proved that the spanning property and the first set of equations imply the three remaining sets of equations.

Our next crucial observation is that the first set of equations is really a formulation about Nullstellensatz in the real category in disguise, in view of an identity of Ozeki and Takeuchi [3]. From this point onwards, we complexified to harness the rich complex algebraic geometry to our advantages, which eventually led to an induction procedure and an estimate on the dimension of certain singular varieties, to verify that the first set of equations and the spanning property always hold on $M_{+}$when $m_{2} \geq 2 m_{1}-1$, where $m_{2}$ is the dimension of the other focal submanifold. Therefore, the isoparametric hypersuface is of FKM-type, on which the Clifford system acts on $M_{+}$, if $m_{2} \geq 2 m_{1}-1$.

The only unsettled cases not handled by the bound $m_{2} \geq 2 m_{1}-1$ are exactly the exceptional ones with multiplicity pairs $(3,4),(4,5),(6,9)$ and $(7,8)$. It appears that handling these exceptional cases in general would entail taking all the four sets of equations into account.

The proof that these four sets of equations characterize an isoparametric 
hypersurface of FKM-type in [2] is an extremely long calculation with remarkable cancellations, which is motivated by the idea that an isoparametric hypersurface is defined by an over-determined system of partial differential equations. Therefore, exterior differentiating the four sets of equations sufficiently many times should gather us enough information for the conclusion on a local scale, which then implies its global counterpart by analyticity. In spite of its elementary nature, the magnitude of the calculation and the surprisingly pleasant cacellations make it desirable to understand the underlying geometric principles.

The purpose of this paper is to give a conceptual, and considerably shorter, proof of the characterization that these four sets of equations are equivalent to that the underlying isoparametric hypersurface is of FKMtype. We first show that the first three sets of equations give rise to a manifold (diffeomorphic to a sphere of dimension $m_{1}$ ) worth of intrinsic isometries of $M_{+}$, whereas the fourth set of equations asserts that these intrinsic isometries extend to ambient isometries of the ambient sphere. We then explore further geometries of $M_{+}$, in conjunction with Ozeki and Takeuchi's expansion formula of the Cartan-Münzner polynomial [3], to verify that the sphere worth of isometries, when extended to ambient isometries, form a round sphere in the space of symmetric matrices. This says precisely that these isometries form a Clifford sphere, and so the isoparametric hypersurface is of FKM-type.

The work would not have been done without the inspiring papers of Ozeki and Takeuchi [3].

\section{§2. Preliminaries}

\subsection{Unit normal bundle of a focal submanifold of an isopara- metric hypersurfaces with four principal curvatures}

Let

$$
\mathbf{x}: M^{n} \longrightarrow S^{n+m+1}
$$

be a submanifold with normal bundle

$$
\mathcal{N}=:\left\{(\mathbf{x}, \mathbf{n}) \in \mathbb{R}^{n+m+2} \times \mathbb{R}^{n+m+2} \mid \mathbf{n} \perp T_{\mathbf{x}} M, \mathbf{n} \perp \mathbf{x}\right\} .
$$

Let $U \mathcal{N}$ be the unit normal bundle of $M$. The Riemannian connection on $M$ splits the tangent bundle of $U \mathcal{N}$ in such a way that the horizontal vectors $X_{u}$ at $u=(x, n) \in U \mathcal{N}$ are the ones satisfying

$$
d \mathbf{n}\left(X_{u}\right) \in T_{\mathbf{x}} M \subset \mathbb{R}^{n+m+2} .
$$


Now let $M^{n}$ be a focal submanifold of an isoparametric hypersurface in $S^{n+m+1}, n=m+2 N$. For each point $(x, n)$ on $U \mathcal{N}$, we let $X_{p}, m+1 \leq$ $p \leq 2 m, X_{\alpha}, 2 m+1 \leq \alpha \leq 2 m+N, X_{\mu}, 2 m+N+1 \leq \mu \leq 2 m+2 N$, be orthonormal basis eigenvectors with eigenvalues $0,1,-1$, respectively, of the shape operator $A_{n}$. Then these eigenvectors can be lifted to the tangent space at $(x, n)$ via the isomorphism between the horizontal distribution at $(x, n)$ and the tangent space to $M$ at $x$. Explicitly, if

$$
\mathbf{x}: M \longrightarrow S^{n+m+1}
$$

is the embedding, then by $(1)$, for $k=0,1,-1$, respectively,

$$
d(k \mathbf{x}+\mathbf{n})(\bar{X})=0
$$

at $(x, n)$ defines exactly the horizontal lift $\bar{X}$ of $X$, when $X$ is an eigenvector of the shape operator $A_{n}$ with eigenvalue $k$. In fact, since

$$
d \mathbf{n}(\bar{X})=-k d \mathbf{x}(\bar{X})=-k X
$$

by (2), we have

$$
\bar{X}=(d \mathbf{x}(\bar{X}), d \mathbf{n}(\bar{X}))=(X,-k X),
$$

so that the tangent space to $U \mathcal{N}$ at $(x, n)$ splits into

$$
\mathbf{V} \oplus \mathbf{H}_{0} \oplus \mathbf{H}_{1} \oplus \mathbf{H}_{-1}
$$

where $\mathbf{V}$ is the vertical space spanned by $\bar{X}_{a}=\left(0, X_{a}\right), a=1, \ldots, m$, $\mathbf{H}_{s}, s=0,1,-1$, are horizontal subspaces spanned by vectors of the form $\bar{X}_{p}=\left(X_{p}, 0\right), \bar{X}_{\alpha}=\left(X_{\alpha},-X_{\alpha}\right), \bar{X}_{\mu}=\left(X_{\mu}, X_{\mu}\right)$, in the $p, \alpha, \mu$ ranges specified above, whose dual frames are $\theta^{a}, \theta^{p}, \theta^{\alpha}, \theta^{\mu}$, respectively.

\subsection{Lie sphere geometry}

Quantitatively, Lie sphere geometry provides an ideal ground for the unit normal bundle geometry. We will refer to the book [1] for details and further references. Consider $\mathbb{R}^{n+m+4}$ with the metric

$$
\langle x, y\rangle:=-x^{0} y^{0}+x^{1} y^{1}+\cdots+x^{n+m+2} y^{n+m+2}-x^{n+m+3} y^{n+m+3}
$$

of signature $(n+m+2,2)$. The equation $\langle x, x\rangle=0$ defines a quadric $Q^{n+m+2}$ of dimension $n+m+2$ in $\mathbb{R} P^{n+m+3}$. A Lie sphere transformation is precisely a projective transformation of $\mathbb{R} P^{n+m+3}$ which maps $Q^{n+m+2}$ 
to itself. To realize the Lie sphere transformation group, consider, similar to an orthonormal frame in the case of an orthogonal group, a Lie frame, which is an ordered set of vectors $Y_{0}, \ldots, Y_{n+m+3}$ in $\mathbb{R}^{n+m+4}$ such that $\left\langle Y_{a}, Y_{b}\right\rangle=h_{a b}$, where

$$
\left(h_{a b}\right):=\left(\begin{array}{ccc}
0 & 0 & -J \\
0 & I_{n+m} & 0 \\
-J & 0 & 0
\end{array}\right),
$$

where $I_{n+m}$ is the identity matrix of the indicated size, $J$ is the $2 \times 2$ matrix with $J_{11}=J_{22}=0$ and $J_{12}=J_{21}=1$. A Lie frame induces a Lie transformation, and vice versa.

The unit tangent bundle of the sphere $S^{n+m+1}$ now naturally identifies with $\Lambda^{2 n+2 m+1}$, the space of dimension $2 n+2 m+1$ of (projective) lines in $Q^{n+m+2}$, via the identification

$$
\lambda:(\mathbf{x}, \mathbf{n}) \longmapsto[(1, \mathbf{x}, 0),(0, \mathbf{n}, 1)],
$$

where the image of the map denotes the line spanned by the two points $[1, \mathbf{x}, 0]$ and $[0, \mathbf{n}, 1]$ in $Q^{n+m+2}$. The unit normal bundle $U \mathcal{N}$ of a focal submanifold $M^{n}$ of an isoparametric hypersurface in $S^{n+m+1}$ therefore inherits a map into $\Lambda^{2 n+2 m+1}$ via (4). In fact one can readily construct a local smooth Lie frame field on $U \mathcal{N}$ as follows. At $(x, n) \in U \mathcal{N}$, we let $X_{a}$ be a choice of orthonormal vertical frame fields, and $X_{p}, X_{\alpha}, X_{\mu}$ be a choice of the respective orthonormal characteristic frame fields of $A_{n}$. Then

$$
\begin{aligned}
& Y_{0}=(1, x, 0), \quad Y_{1}=(0, n, 1), \\
& Y_{a}=\left(0, X_{a}, 0\right), \quad Y_{p}=\left(0, X_{p}, 0\right), \\
& Y_{\alpha}=\left(0, X_{\alpha}, 0\right), \quad Y_{\mu}=\left(0, X_{\mu}, 0\right), \\
& Y_{n+m+2}=\left(0,-\frac{1}{2} n, \frac{1}{2}\right), \quad Y_{n+m+3}=\left(\frac{1}{2},-\frac{1}{2} x, 0\right),
\end{aligned}
$$

is a Lie frame field. We set

$$
d Y_{j}=\sum_{i} \omega_{j}^{i} Y_{i}
$$

Then the Maurer-Cartan equations applied to $\left(\omega_{j}^{i}\right)$, which lies in the Lie algebra of the Lie sphere group, imply

$$
d \omega_{j}^{i}=-\sum_{k} \omega_{k}^{i} \wedge \omega_{j}^{k} .
$$


An easy calculation shows that

$$
\omega_{0}^{0}=\omega_{1}^{1}=\omega_{0}^{1}=\omega_{1}^{0}=0,
$$

and

$$
\begin{array}{ll}
\omega_{1}^{a}=\theta^{a}, & \omega_{0}^{p}=\theta^{p}, \\
\omega_{0}^{\alpha}=\theta^{\alpha}, & \omega_{0}^{\mu}=\theta^{\mu},
\end{array}
$$

where $\theta^{a}, \theta^{p}, \theta^{\alpha}, \theta^{\mu}$ are the dual forms on $U \mathcal{N}$ introduced in the preceding section. Furthermore, we have

$$
\begin{aligned}
& \omega_{0}^{a}=0, \quad \omega_{1}^{p}=0, \\
& \omega_{0}^{\alpha}+\omega_{1}^{\alpha}=0, \quad-\omega_{0}^{\mu}+\omega_{1}^{\mu}=0 .
\end{aligned}
$$

Now, on $U \mathcal{N}$ we set

$$
\left\langle d X_{j}, X_{i}\right\rangle=\omega_{j}^{i}:=\sum_{k} F_{j k}^{i} \theta^{k}
$$

where $i, j, k$ run through the $a, p, \alpha, \mu$ ranges. Note that $F_{j k}^{i}=-F_{i k}^{j}$. Differentiating (8) with (5), (6) and (7) in mind, we obtain that $F_{j k}^{i}=0$ whenever exactly two of the indices come from the same range. Moreover,

$$
\begin{aligned}
& F_{a \alpha}^{p}=-F_{\alpha a}^{p}=F_{p a}^{\alpha}=F_{a p}^{\alpha}, \\
& F_{a \mu}^{p}=F_{\mu a}^{p}=-F_{p a}^{\mu}=F_{a p}^{\mu}, \\
& F_{p \mu}^{\alpha}=2 F_{\mu p}^{\alpha}=-2 F_{\alpha p}^{\mu}=-F_{p \alpha}^{\mu}, \\
& F_{a \mu}^{\alpha}=2 F_{\mu a}^{\alpha}=-2 F_{\alpha a}^{\mu}=F_{a \alpha}^{\mu} .
\end{aligned}
$$

In particular, (9) and (10) assert that

$$
\begin{aligned}
F_{p a}^{\alpha} & =-\left\langle A_{X_{a}}\left(X_{\alpha}\right), X_{p}\right\rangle, \\
F_{p a}^{\mu} & =\left\langle A_{X_{a}}\left(X_{\mu}\right), X_{p}\right\rangle, \\
F_{\alpha a}^{\mu} & =\frac{1}{2}\left\langle A_{X_{a}}\left(X_{\alpha}\right), X_{\mu}\right\rangle .
\end{aligned}
$$

We will see the meaning of $F_{\alpha p}^{\mu}$ in the next section. Note that (5) through (10) also imply the structural equations (with Einstein summation convention)

$$
\begin{aligned}
& d \theta^{a}=-\omega_{b}^{a} \wedge \theta^{b}-F_{p a}^{\alpha} \theta^{p} \wedge \theta^{\alpha}-F_{p a}^{\mu} \theta^{p} \wedge \theta^{\mu}-4 F_{\alpha a}^{\mu} \theta^{\alpha} \wedge \theta^{\mu}, \\
& d \theta^{p}=-\omega_{q}^{p} \wedge \theta^{q}+F_{p a}^{\alpha} \theta^{a} \wedge \theta^{\alpha}+F_{p a}^{\mu} \theta^{a} \wedge \theta^{\mu}+4 F_{\alpha p}^{\mu} \theta^{\alpha} \wedge \theta^{\mu}, \\
& d \theta^{\alpha}=-\omega_{\beta}^{\alpha} \wedge \theta^{\beta}-F_{p a}^{\alpha} \theta^{a} \wedge \theta^{p}+F_{\alpha a}^{\mu} \theta^{a} \wedge \theta^{\mu}-F_{\alpha p}^{\mu} \theta^{p} \wedge \theta^{\mu}, \\
& d \theta^{\mu}=-\omega_{\nu}^{\mu} \wedge \theta^{\nu}-F_{p a}^{\mu} \theta^{a} \wedge \theta^{p}-F_{\alpha a}^{\mu} \theta^{a} \wedge \theta^{\alpha}+F_{\alpha p}^{\mu} \theta^{p} \wedge \theta^{\alpha} .
\end{aligned}
$$




\section{$\S 3 . \quad$ The symmetries}

Consider the natural isometry

$$
T:(p, q) \longmapsto(q, p)
$$

from $\mathbb{R}^{n+m+2} \times \mathbb{R}^{n+m+2}$ into itself.

Proposition 1. Retaining the preceding notations, $T$ leaves $U \mathcal{N}$ invariant in the case of four principal curvatures.

Proof. The exponential map

$$
\exp :(x, \mathbf{n}(x)) \longmapsto p=\cos t x+\sin t \mathbf{n}(x)
$$

of the sphere $S^{n+m+1}$ maps $U \mathcal{N}$ to an isoparametric hypersurface $M_{t}$ in general, and returns to the focal submanifold at $t=\pi / 2$, at which $p=$ $\mathbf{n}(x)$ and the derivative of the map is $-x$, which is normal to the focal submanifold.

Corollary 1. Any local section $s: M \rightarrow U \mathcal{N}, s: x \mapsto(x, Q(x))$, gives rise to a local map from $M$ into itself.

Proof. Let $\pi: U \mathcal{N} \longrightarrow M$ be the projection. Consider the local map $\pi \circ T \circ s: M \rightarrow M$, which is just the map

$$
g: x \longmapsto Q(x) .
$$

Since $T$ is an isometry on $U \mathcal{N}$, we next understand its tangent map. Note that by $(3)$, at $(x, n)$, we have the orthonormal frame $\left(0, X_{a}\right),\left(X_{p}, 0\right)$, $\left(X_{\alpha},-X_{\alpha}\right),\left(X_{\mu}, X_{\mu}\right)$ dual to $\theta^{a}, \theta^{p}, \theta^{\alpha}, \theta^{\mu}$. By the fact that $T$ is a linear map interchanging the two coordinates, we obtain

Proposition 2.

$$
\begin{aligned}
T_{*} & :\left(0, X_{a}\right) \longmapsto\left(X_{a}, 0\right), \\
& :\left(X_{p}, 0\right) \longmapsto\left(0, X_{p}\right), \\
& :\left(X_{\alpha},-X_{\alpha}\right) \longmapsto\left(-X_{\alpha}, X_{\alpha}\right), \\
& :\left(X_{\mu}, X_{\mu}\right) \longmapsto\left(X_{\mu}, X_{\mu}\right)
\end{aligned}
$$

from the tangent space at $(x, n)$ to the tangent space at $(n, x)$ on $U \mathcal{N}$, so that $T_{*}$ interchanges the $E_{a}$ and $E_{p}$ distributions and fixes the $E_{\alpha}$ and $E_{\mu}$ distributions. 
It follows immediately from the proposition the following.

Corollary 2. $F_{\alpha p}^{\mu}$ at $(x, n) \in U \mathcal{N}$ is exactly $F_{\alpha a}^{\mu}$ at $(n, x) \in U \mathcal{N}$.

Consider now the local map

$$
g: x \longmapsto Q(x)
$$

arising from a local section $s: M \rightarrow U \mathcal{N}, s: M \mapsto(x, Q(x))$, in Corollary 1 . We ask when $g$ is a local isometry on the focal submanifold $M$.

Lemma 1. Retain the notations in Corollary 1 and let $X_{p}, X_{\alpha}$, and $X_{\mu}$ as before be appropriate orthonormal eigenvectors for the shape operator $A_{Q(x)}$. Then $g$ is a local isometry of $M$ if and only if $s_{*}$ maps $X_{\alpha}$ and $X_{\mu}$ to their horizontal lifts at $(x, Q(x))$, and maps $X_{p}$ to $\left(X_{p}, V_{p}\right)$ such that $X_{p} \mapsto V_{p}$ is an isometry.

Proof. Let $s_{*}\left(X_{p}\right)=\left(X_{p}, V_{p}\right), s_{*}\left(X_{\alpha}\right)=\left(X_{\alpha},-X_{\alpha}+V_{\alpha}\right)$, and $s_{*}\left(X_{\mu}\right)=$ $\left(X_{\mu}, X_{\mu}+V_{\mu}\right)$. That is, we break the three images under $s_{*}$ into horizontal and vertical components. By the very definition of $g$ we see

$$
\begin{aligned}
& g_{*}: X_{p} \longmapsto V_{p}, \\
& g_{*}: X_{\alpha} \longmapsto V_{\alpha}-X_{\alpha}, \\
& g_{*}: X_{\mu} \longmapsto V_{\mu}+X_{\mu} .
\end{aligned}
$$

Since the vertical components $V_{p}, V_{\alpha}$ and $V_{\mu}$ are all perpendicular to $X_{p}$, $X_{\alpha}$ and $X_{\mu}$, we see $g_{*}$ is a local isometry if and only if $V_{\alpha}=V_{\mu}=0$.

At each point $u=(x, n)$ of $U \mathcal{N}$, we set, respectively, $\mathbf{E}_{a}, \mathbf{E}_{p}, \mathbf{E}_{\alpha}, \mathbf{E}_{\mu}$ to be the vertical space at $u$ and the three horizontal eigenspaces of the shape operator $A_{n}$ with eigenvalue $0,1,-1$ pulled back to the horizontal space at $u$. In light of Lemma 1, we assign smoothly an isometry $O_{u}$ from $\mathbf{E}_{p}$ to $\mathbf{E}_{a}$. Let

$$
\mathbf{F}_{p}=\left\{X_{p}+O_{u}\left(X_{p}\right) \mid X_{p} \in \mathbf{E}_{p}\right\}
$$

at $u$ and consider the distribution

$$
\boldsymbol{\Delta}_{u}=\mathbf{F}_{p} \oplus \mathbf{E}_{\alpha} \oplus \mathbf{E}_{\mu}
$$

If this distribution is integrable, then according to Lemma 1, each leaf $Q(x)$ will induce an isometry on $M$. In accordance, we seek to find a necessary 
and sufficient condition for the distribution to be integrable. We can arrange so that

$$
-X_{p-m}=O_{u}\left(X_{p}\right)
$$

Remark 1. Before we proceed, let us look at the isoparametric hypersurfaces of FKM-type [4]. Let $P_{0}, \ldots, P_{m}$ be a Clifford system on $\mathbb{R}^{2 l}$, which are orthogonal symmetric operators on $\mathbb{R}^{2 l}$ satisfying

$$
P_{i} P_{j}+P_{j} P_{i}=2 \delta_{i j} I, \quad i, j=0, \ldots, m .
$$

The 4th degree homogeneous polynomial

$$
F(x)=|x|^{4}-2 \sum_{i=0}^{m}\left(\left\langle P_{i}(x), x\right\rangle\right)^{2}
$$

is the Cartan-Münzner polynomial, so that $F^{-1}(t),-1<t<1$, on the sphere is a 1-parameter family of isoparametric hypersurfaces whose focal submanifolds are $M_{ \pm}=F^{-1}( \pm 1)$.

$M_{+}$is the variety carved out by the quadrics $\left\langle P_{i}(x), x\right\rangle=0, i=$ $0, \ldots, m$, whose normal bundle at $x$ is spanned by $P_{0}(x), \ldots, P_{m}(x)$. If we set $Q:=\sum_{j} a^{j} P_{j}$, where $\sum_{j}\left(a^{j}\right)^{2}=1$, then $\left\{Q(x): x \in M_{+}\right\}$is a leaf in the unit normal bundle of $M_{+}$. These leaves, as $Q$ varies, give rise to an integrable distribution $\boldsymbol{\Delta}$ of the sort we are considering. In fact, at $x$ the 0 eigenspace of the shape operator $A_{Q(x)}$ is spanned by $P Q(x)$, where $P \perp Q$ for all $P$. Therefore, a typical vector in the 0-eigenspace, say, $X_{p}:=P Q(x)$, will be mapped via $Q$ to $-P(x)$ in the normal space at $x$, which we designate as $-X_{p-m}$. That is, $-X_{p-m}=Q\left(X_{p}\right)$, which is compatible with (16).

Proposition 3. $\boldsymbol{\Delta}$ is involutive if and only if

$$
\begin{aligned}
F_{\alpha p}^{\mu} & =F_{\alpha p-m}^{\mu}, \\
F_{a+m b}^{\alpha} & =-F_{b+m a}^{\alpha}, \\
F_{a+m b}^{\mu} & =-F_{b+m a}^{\mu} .
\end{aligned}
$$

Proof. (Sketch) $\boldsymbol{\Delta}$ is the kernel of

$$
\theta^{a}+\theta^{a+m}
$$

for all $a$, which we differentiate while invoke (12). 
Proposition 4. When $\boldsymbol{\Delta}$ is involutive, the isometries $g$ induced by the leaves of $\boldsymbol{\Delta}$ extend to ambient isometries in $S^{n+m+1}$ if and only if

$$
\omega_{a}^{b}-\omega_{a+m}^{b+m}=\sum_{p} L_{b a}^{p}\left(\theta^{p-m}+\theta^{p}\right) .
$$

for some $L_{b a}^{p}$. In particular, the unit normal bundle of $M_{+}$of an isoparametric hypersurface of FKM-type satisfies (17) through (20).

Proof. We will show that each $g_{*}$ leaves the 2 nd fundamental form and the normal connection form invariant, from which the rigidity follows [6].

Recall from Lemma 1 that we let $X_{\alpha}, X_{p}, X_{\mu}$ be respective orthonormal characteristic vecotr fields of $A_{Q(x)}$ in $M$, and let $X_{a}$ be orthonormal normal vector fields perpendicular to the normal vector $Q(x)$ at $x$ in $M$; in fact, $X_{a}, X_{p}, X_{\alpha}, X_{\mu}$ form a Lie frame field over the section $s$. Recall that $g: x \mapsto Q(x)$ is induced from the leaf $s: x \mapsto(x, Q(x))$, where

$$
\begin{aligned}
& s_{*}\left(X_{p}\right)=\left(X_{p},-X_{p-m}\right) . \\
& s_{*}\left(X_{\alpha}\right)=\left(X_{\alpha},-X_{\alpha}\right), \\
& s_{*}\left(X_{\mu}\right)=\left(X_{\mu}, X_{\mu}\right),
\end{aligned}
$$

by the definition of the distribution $\Delta$. So we have from (13), (14) and (15)

$$
\begin{aligned}
& g_{*}\left(X_{p}\right)=-X_{p-m} \\
& g_{*}\left(X_{\alpha}\right)=-X_{\alpha} \\
& g_{*}\left(X_{\mu}\right)=X_{\mu} .
\end{aligned}
$$

To keep our notation straight, we let

$$
\begin{aligned}
Y_{-1}(g(x)) & :=Q(x), \\
Y_{0}(g(x)) & :=x, \\
Y_{a}(g(x)) & :=-X_{a+m}(x), \\
Y_{p}(g(x)) & :=-X_{p-m}(x), \\
Y_{\alpha}(g(x)) & :=-X_{\alpha}(x), \\
Y_{\mu}(g(x)) & :=X_{\mu}(x) .
\end{aligned}
$$

We set $X_{-1}(x):=x, X_{0}(x):=Q(x) . Y_{-1}, Y_{0}$ and $Y_{a}$ are normal, and $Y_{p}$, $Y_{\alpha}, Y_{\mu}$ are tangent to $M$ at $g(x)$, in contrast to $X_{-1}, X_{0}$ and $X_{a}$ being 
normal and $X_{p}, X_{\alpha}, X_{\mu}$ being tangent to $M$ at $x$, when we regard $M$ as a submanifold of $\mathbb{R}^{n+m+2}$. We therefore have set up a normal bundle isomorphism

$$
\Psi: X_{a} \longmapsto Y_{a}, \quad-1 \leq a \leq m,
$$

between the normal bundle of $M$ over $x$ and the normal bundle of $M$ over $g(x)$ covering $g_{*}$.

The 2 nd fundamental form at $x$ is

$$
S(X, Y)=-\sum_{a}\left\langle d X_{a}(X), Y\right\rangle X_{a}
$$

for $X, Y \in T M, a=-1, \ldots, m$, and is

$$
\Pi(X, Y)=-\sum_{a}\left\langle d Y_{a}(X), Y\right\rangle Y_{a}
$$

at $g(x)$. In view of $(21),(22),(23)$,

$$
\begin{aligned}
\Pi\left(g_{*}\left(X_{\alpha}\right), g_{*}\left(X_{\mu}\right)\right) & =-\sum_{a \geq 1}\left\langle d Y_{a}\left(g_{*}\left(X_{\alpha}\right)\right), g_{*}\left(X_{\mu}\right)\right\rangle Y_{a} \\
& =-\sum_{a \geq 1}\left\langle-d X_{a+m}\left(X_{\alpha}\right), X_{\mu}\right\rangle\left(-X_{a+m}\right) \\
& =-\sum_{a \geq 1}\left\langle d X_{a+m}\left(s_{*}\left(X_{\alpha}\right)\right), X_{\mu}\right\rangle X_{a+m} \\
& =-\sum_{a \geq 1} \sum_{t} F_{a+m t}^{\mu} \theta^{t}\left(s_{*}\left(X_{\alpha}\right)\right) X_{a+m} \\
& =-\sum_{a \geq 1} \sum_{t} F_{a+m t}^{\mu} \theta^{t}\left(\left(X_{\alpha},-X_{\alpha}\right)\right) X_{a+m} \\
& =-\sum_{a \geq 1} F_{a+m \alpha}^{\mu} X_{a+m},
\end{aligned}
$$

where the third equality follows from the fact that the frames $X_{\alpha}$ are indeed smoothly defined as part of a Lie frame over the section $s$, so that the exterior differentiation can be conducted over $s$ with respect to $s_{*}\left(X_{\alpha}\right)$ that covers $X_{\alpha}$. Likewise,

$$
\begin{aligned}
\Psi\left(S\left(X_{\alpha}, X_{\mu}\right)\right) & =-\sum_{a \geq 1}\left\langle d X_{a}\left(X_{\alpha}\right), X_{\mu}\right\rangle \Psi\left(X_{a}\right) \\
& =-\sum_{a \geq 1} F_{a \alpha}^{\mu}\left(-X_{a+m}\right)=\sum_{a \geq 1} F_{a \alpha}^{\mu} X_{a+m}
\end{aligned}
$$


So they are equal by (10) and (17). We remark that $a=-1,0$ do not appear in the above equalities because, for instance,

$$
\begin{aligned}
\left\langle d Y_{0}\left(g_{*}\left(X_{\alpha}\right)\right), g_{*}\left(X_{\mu}\right)\right\rangle Y_{0} & =\left\langle d x\left(X_{\alpha}\right), X_{\mu}\right\rangle x \\
& =\left\langle X_{\alpha}, X_{\mu}\right\rangle x=0 \\
& =\Psi\left(S\left(X_{\alpha}, X_{\mu}\right)\right) .
\end{aligned}
$$

In the same vein, for $a=1, \ldots, m$,

$$
\begin{aligned}
\Pi\left(g_{*}\left(X_{p}\right), g_{*}\left(X_{\alpha}\right)\right) & =-\sum_{a \geq 1}\left\langle d Y_{a}\left(g_{*}\left(X_{p}\right)\right), g_{*}\left(X_{\alpha}\right)\right\rangle Y_{a} \\
& =-\sum_{a \geq 1}\left\langle-d X_{a+m}\left(X_{p}\right),-X_{\alpha}\right\rangle\left(-X_{a+m}\right) \\
& =\sum_{a \geq 1}\left\langle d X_{a+m}\left(s_{*}\left(X_{p}\right)\right), X_{\alpha}\right\rangle X_{a+m} \\
& =\sum_{a \geq 1} \sum_{t} F_{a+m, t}^{\alpha} \theta^{t}\left(s_{*}\left(X_{p}\right)\right) X_{a+m} \\
& =\sum_{a \geq 1} \sum_{t} F_{a+m, t}^{\alpha} \theta^{t}\left(\left(X_{p}, 0\right)+\left(0,-X_{p-m}\right)\right) X_{a+m} \\
& =\sum_{a \geq 1}\left(F_{a+m, p}^{\alpha}-F_{a+m, p-m}^{\alpha}\right) X_{a+m} \\
& =-\sum_{a \geq 1} F_{a+m, p-m}^{\alpha} X_{a+m},
\end{aligned}
$$

where we invoke the fact that $s_{*}\left(X_{p}\right)=\left(X_{p}, 0\right)+\left(0,-X_{p-m}\right)$ with $\left(X_{p}, 0\right)$ horizontal and $\left(0,-X_{p-m}\right)$ vertical. Likewise,

$$
\begin{aligned}
\Psi\left(S\left(X_{p}, X_{\alpha}\right)\right) & =-\sum_{a \geq 1}\left\langle d X_{a}\left(X_{p}\right), X_{\alpha}\right\rangle \Psi\left(X_{a}\right) \\
& =-\sum_{a \geq 1} F_{a p}^{\alpha}\left(-X_{a+m}\right)=\sum_{a \geq 1} F_{a p}^{\alpha} X_{a+m} .
\end{aligned}
$$

So they are equal by (18). Similar identities hold for other pairs of vectors. In short,

$$
\Pi \circ g_{*}=\Psi \circ S .
$$

The normal connection form is

$$
D X_{a}=\sum_{b} \Lambda_{a}^{b} X_{b},
$$


where $\Lambda_{a}^{b}=\left\langle d X_{a}, X_{b}\right\rangle$ at $x$ and is

$$
\bar{D} Y_{a}=\sum_{b} \Theta_{a}^{b} Y_{b}
$$

where $\Theta_{a}^{b}=\left\langle d Y_{a}, Y_{b}\right\rangle$ at $g(x)$. We next establish

$$
g^{*} \Theta_{a}^{b}=\Lambda_{a}^{b}
$$

that is,

$$
\bar{D}_{g_{*}(V)}(\Psi(\zeta))=\Psi\left(D_{V}(\zeta)\right)
$$

(25) and (26) will establish the rigidity. Now

$$
\begin{aligned}
g^{*} \Theta_{a}^{b}\left(X_{\alpha}\right) & =\left\langle-d X_{a+m}\left(X_{\alpha}\right),-X_{b+m}\right\rangle \\
& =F_{a+m, \alpha}^{b+m}=0
\end{aligned}
$$

while $\Lambda_{a}^{b}\left(X_{\alpha}\right)=0$ similarly. On the other hand,

$$
\begin{aligned}
g^{*} \Theta_{a}^{b}\left(X_{p}\right) & =\left\langle d Y_{a}\left(g_{*}\left(X_{p}\right)\right), Y_{b}\right\rangle \\
& =\left\langle-d X_{a+m}\left(X_{p}\right),-X_{b+m}\right\rangle \\
& =\omega_{a+m}^{b+m}\left(s_{*}\left(X_{p}\right)\right),
\end{aligned}
$$

while

$$
\begin{aligned}
\Lambda_{a}^{b}\left(X_{p}\right) & =\left\langle d X_{a}\left(X_{p}\right), X_{b}\right\rangle \\
& =\left\langle d X_{a}\left(s_{*}\left(X_{p}\right)\right), X_{b}\right\rangle \\
& =\omega_{a}^{b}\left(s_{*}\left(X_{p}\right)\right) .
\end{aligned}
$$

Therefore they are equal if and only if $\omega_{a}^{b}-\omega_{a+m}^{b+m}$ annihilates $s_{*}\left(X_{p}\right)$, if and only if it annihilates the distribution $\Delta$ because it automatically annihilates the horizontal $s_{*}\left(X_{\alpha}\right)$ and $s_{*}\left(X_{\mu}\right)\left(F_{j k}^{i}=0\right.$ if exactly two indices are from the same range), if and only if

$$
\omega_{a}^{b}-\omega_{a+m}^{b+m}=\sum_{p} L_{b a}^{p}\left(\theta^{p-m}+\theta^{p}\right)
$$

for some $L_{b a}^{p}$ because $\theta^{p-m}+\theta^{p}$, for all $p$, form the dual of $\Delta$. 


\section{§4. The focal submanifold $M_{+}$is a real affine variety}

Conversely, assuming now that (17) through (20) hold true, we will establish that the isoparametric hypersurface is of FKM-type.

By Proposition 4 , each leaf now is of the form $(x, Q \cdot x)$ for some constant orthogonal matrix $Q$, so that in fact it induces a global isometry $x \mapsto Q \cdot x$ on $M$. ("." denotes matrix multiplication.) Note also that since $Q \cdot x$ is a normal vector at $x \in M$, we have $\langle Q \cdot x, x\rangle=0$. In fact we have an $S^{m}$-worth of such $Q$ 's because there is a leaf through each point of a fiber of $U \mathcal{N}$; let the set of the $S^{m}$-worth of $Q$ 's be denoted by $\mathcal{C}$. Now $\mathcal{C}$ begins to look like the Clifford sphere. One needs to establish next the Clifford properties of the $Q$ 's in $\mathcal{C}$.

We first show that

$$
Q^{2}=I d
$$

for all $Q$ in $\mathcal{C}$. Retaining all the previous notations, we see that $Q$ is exactly $\Psi$ in (24). Hence $x+Q \cdot x, X_{a}-X_{a+m}, X_{\mu}$ are eigenvectors of $Q$ with eigenvalue 1 , while $x-Q \cdot x, X_{a}+X_{a+m}, X_{\alpha}$ are eigenvectors of $Q$ with eigenvalue -1 , which implies that $Q$ is symmetric. So $Q^{2}=I d$ because $Q$ is also orthogonal.

Definition 1. $M_{+}$is the focal submanifold satisfying (17) through (20).

LEMMA 2.

$$
M_{+}=\left\{x \in S^{n+m+1}:\langle Q \cdot x, x\rangle=0, \text { all } Q \in \mathcal{C}\right\},
$$

so that $M_{+}$is a real affine variety.

Proof. For $x$ in $M_{+}, Q \cdot x$ is a normal vector for any $Q \in \mathcal{C}$. So clearly $\langle Q \cdot x, x\rangle=0$. Conversely, the sphere $S^{n+m+1}$ is covered by the exponential map

$$
\exp :(t, x, P) \longmapsto y=:(\cos t) x+(\sin t) P \cdot x
$$

with $x$ in $M_{+}$and $P$ in $\mathcal{C}$. We ask when $y$ satisfies $\langle Q \cdot y, y\rangle=0$ for all $Q$. This is equivalent to, upon expansion, the condition

$$
\sin 2 t\langle P \cdot x, Q \cdot x\rangle=0
$$

for all $Q$ in $\mathcal{C}$. When picking $Q$ to be $P$, we see by $P^{2}=I d$ that this is in turn equivalent to $\sin 2 t=0$. In other words, $t=0, \pi / 2$, or $\pi$, which implies that $y$ lies in $M_{+}$. 


\section{$\S 5$. More geometry of $M_{+}$}

Fix a point $e \in M_{+}$. We have the decomposition

$$
\mathbb{R}^{2+m+n}=\mathbb{R} e \oplus T \oplus N
$$

where $T$ and $N$ are the tangent and normal spaces of $M_{+}$at $e$. We write a typical element in $\mathbb{R}^{2+m+n}$ as

$$
t e+y+w,
$$

where $t \in \mathbb{R}, y \in T$, and $w \in N$, with respect to the decomposition. We will from now on coordinatize $\mathbb{R}^{2+m+n}$ this way. Clearly, $t= \pm 1, y=w=0$ are two points on $M_{+} \cdot\left(M_{+}\right.$is diametrically symmetric.) Let

$$
C M_{+}:=\left\{r x: r \in \mathbb{R}, x \in M_{+}\right\}
$$

be the cone over $M_{+}$.

Convention 1. Pick $P_{0}, \ldots, P_{m} \in \mathcal{C}$ such that $P_{0} \cdot e, \ldots, P_{m} \cdot e$ are orthonormal. This is possible since the map $\mathcal{C} \rightarrow U_{e} \mathcal{N}$ given by $P \mapsto P \cdot e$ is a diffeomorphism. Henceforth, we refer to $P_{0}, \ldots, P_{m}$ as such a choice in $\mathcal{C}$.

Remark 2. All identities to be derived below will not be hard to verify if $\mathcal{C}$ is a round sphere, which will be our end result. However, at this point $\mathcal{C}$ is only diffeomorphic to a sphere. What is remarkable is that the identities remain true under the weaker condition that $\mathcal{C}$ is a diffeomorphic sphere.

Lemma 3. Let $t_{0} e+y_{0}+w_{0} \in C M_{+}$. Then the line te $+y_{0}+w_{0}$ parametrized by $t$ intersects $C M_{+}$in exactly one point if $w_{0} \neq 0$.

Proof. First, note that $\left\langle P_{i} \cdot e, e\right\rangle=\left\langle P_{i} \cdot e, y_{0}\right\rangle=0$, since $P_{i} \cdot e$ is a normal vector to $M_{+}$at $e$. Furthermore, $\left\langle P_{i} \cdot w_{0}, w_{0}\right\rangle=0$ because $w_{0} /\left|w_{0}\right| \in M_{+}$ as well by Proposition 1 . It follows that for $0 \leq i \leq m$, we have

$$
\begin{aligned}
0 & =\left\langle P_{i} \cdot\left(t e+y_{0}+w_{0}\right), t e+y_{0}+w_{0}\right\rangle \\
& =\left\langle P_{i} \cdot y_{0}, y_{0}\right\rangle+2 t\left\langle P_{i} \cdot e, w_{0}\right\rangle+2\left\langle P_{i} \cdot y_{0}, w_{0}\right\rangle
\end{aligned}
$$

for $t e+y_{0}+w_{0} \in C M_{+}$. Since $P_{i} \cdot e, i=0, \ldots, m$, form an orthonormal basis for the normal space $N$ of $M_{+}$at $e$, if we set

$$
w_{i}:=\left\langle P_{i} \cdot e, w_{0}\right\rangle
$$


we obtain

$$
\sum_{i} w_{i}^{2}=\left|w_{0}\right|^{2}
$$

Multiplying through (28) by $w_{i}$ and summing up over $i$, we obtain

$$
2 t\left|w_{0}\right|^{2}=-\sum_{i=0}^{m} w_{i}\left\langle P_{i} \cdot y_{0}, y_{0}\right\rangle-2 \sum_{i=0}^{m} w_{i}\left\langle P_{i} \cdot y_{0}, w_{0}\right\rangle .
$$

If $w_{0} \neq 0$, then there is only one solution for $t$.

We record that, along the normal vector $P \cdot e$, we have

$$
\left\langle S\left(v_{1}, v_{2}\right), P \cdot e\right\rangle=-\left\langle P \cdot v_{1}, v_{2}\right\rangle,
$$

where $P \in \mathcal{C}, S$ is the 2 nd fundamental form and $v_{1}$ and $v_{2}$ are two tangent vectors to $M_{+}$at $e$. That is, we have

$$
A_{P \cdot e}(v)=-(P \cdot v)^{T}
$$

where the upper script $T$ denotes orthogonal projection onto the tangential component at $e$ for an tangent vector $v$. The identity is true because $P \cdot x$, as $x$ varies around $e$ in $M_{+}$, is a normal vector field, whose derivative at $e$ gives (31). For notational ease, we set

$$
p_{i}:=-\left\langle P_{i} \cdot y_{0}, y_{0}\right\rangle
$$

Corollary 3. Let $t_{0} e+y_{0}+w_{0} \in C M_{+}, w_{0} \neq 0$. Then $t_{0}$ is the double root of the quadratic polynomial (in $t$ )

$$
\begin{array}{r}
4\left|w_{0}\right|^{2} t^{2}+4\left(\sum_{i=0}^{m}-p_{i} w_{i}+2 \sum_{i=0}^{m} w_{i}\left\langle P_{i} \cdot y_{0}, w_{0}\right\rangle\right) t \\
+\sum_{i=0}^{m} p_{i}^{2}-4 p_{i}\left\langle P_{i} \cdot y_{0}, w_{0}\right\rangle+4\left\langle P_{i} \cdot y_{0}, w_{0}\right\rangle^{2}=0 .
\end{array}
$$

Proof. Squaring (28) and summing over $i$, we obtain the polynomial for which $t_{0}$ is a root. Conversely, suppose $t$ is a root of the polynomial. Tracing backwards, we obtain (28) and so (29). This implies that $t=t_{0}$. 
Now we compare (33) with the equation derived in [3]. Let $F$ be the 4th degree homogeneous Cartan-Münzner polynomial. Then

$$
\begin{aligned}
F(t e+y+w)=t^{4} & +\left(2|y|^{2}-6|w|^{2}\right) t^{2}+8\left(\sum_{i=0}^{m} p_{i} w_{i}\right) t \\
& +|y|^{4}-6|y|^{2}|w|^{2}+|w|^{4}-2 \sum_{i=0}^{m} p_{i}^{2}+8 \sum_{i=0}^{m} q_{i} w_{i} \\
& +2 \sum_{i, j=0}^{m}\left\langle\nabla p_{i}, \nabla p_{j}\right\rangle w_{i} w_{j}
\end{aligned}
$$

where $q_{i}(y), i=0, \ldots, m$, are some cubic homogeneous polynomials in $y$; in fact, they are the 3rd fundamental forms of $M_{+}$.

Lemma 4. $t_{0} e+y_{0}+w_{0} \in C M_{+}, w_{0} \neq 0$. Then $t_{0}$ is the double root of the quadratic polynomial (in $t$ )

$$
4\left|w_{0}\right|^{2} t^{2}-4\left(\sum_{i=0}^{m} p_{i} w_{i}\right) t+\sum_{i=0}^{m}\left(p_{i}^{2}-4 q_{i} w_{i}\right)+4\left|w_{0}\right|^{2}\left|\left(P \cdot y_{0}\right)^{\perp}\right|^{2}=0
$$

where $P \in \mathcal{C}$ is such that

$$
P \cdot e=\sum_{i=0}^{m} \frac{w_{i}}{\left|w_{0}\right|} P_{i} \cdot e\left(=w_{0} /\left|w_{0}\right|\right)
$$

and $\perp$ denotes the orthogonal projection onto the normal space $N$ to $M_{+}$ at $e$.

$$
\text { Proof. }
$$

$$
\begin{aligned}
f & :=F\left(t e+y_{0}+w_{0}\right)-\left|t e+y_{0}+w_{0}\right|^{4} \\
& =F\left(t e+y_{0}+w_{0}\right)-\left(t^{2}+\left|y_{0}\right|^{2}+\left|w_{0}\right|^{2}\right)^{2}=0
\end{aligned}
$$

if $t e+y_{0}+w_{0} \in C M_{+}$, because $F(x)=1$ for $x \in M_{+} ; f$ is a 2 nd order polynomial in $t$ by (34). The only messy term in $f$ is the one involving $\nabla p_{i}$ in (34). However, since

$$
\nabla p_{i}=-2\left(P_{i} \cdot y_{0}\right)^{T}
$$


we have, in view of (31),

$$
\begin{aligned}
& \sum_{i, j=0}^{m}\left\langle\nabla p_{i}, \nabla p_{j}\right\rangle w_{i} w_{j} \\
& =4 \sum_{i, j=0}^{m}\left\langle\left(P_{i} \cdot y_{0}\right)^{T},\left(P_{j} \cdot y_{0}\right)^{T}\right\rangle w_{i} w_{j} \\
& =4 \sum_{i, j=0}^{m}\left\langle A_{P_{i} \cdot e}\left(y_{0}\right), A_{P_{j} \cdot e}\left(y_{0}\right)\right\rangle w_{i} w_{j} \\
& =4\left|w_{0}\right|^{2}\left\langle A_{P \cdot e}\left(y_{0}\right), A_{P \cdot e}\left(y_{0}\right)\right\rangle \\
& =4\left|w_{0}\right|^{2}\left\langle\left(P \cdot y_{0}\right)^{T},\left(P \cdot y_{0}\right)^{T}\right\rangle \\
& =4\left|w_{0}\right|^{2}\left\langle P \cdot y_{0}, P \cdot y_{0}\right\rangle-4\left|w_{0}\right|^{2}\left\langle\left(P \cdot y_{0}\right)^{\perp},\left(P \cdot y_{0}\right)^{\perp}\right\rangle \\
& =4\left|w_{0}\right|^{2}\left|y_{0}\right|^{2}-4\left|w_{0}\right|^{2}\left|\left(P \cdot y_{0}\right)^{\perp}\right|^{2},
\end{aligned}
$$

due to the fact that $P$ is orthogonal. Hence $t_{0}$ is a root of $f$ as a polynomial of $t$.

Conversely, suppose $t$ is a root of the polynomial $f$. Then $t e+y_{0}+w_{0}$ must belong to $C M_{+}$. Thus by Lemma 4 , we have $t=t_{0}$.

Henceforth, we drop the subscript 0 from $y_{0}$ and $w_{0}$ for notational ease.

Corollary 4.

$$
\sum_{i=0}^{m} w_{i}\left\langle P_{i} \cdot y, w\right\rangle=0
$$

and

$$
-\sum_{i=0}^{m} p_{i}\left\langle P_{i} \cdot y, w\right\rangle+\sum_{i=0}^{m}\left\langle P_{i} \cdot y, w\right\rangle^{2}=-\sum_{i=0}^{m} q_{i} w_{i}+|w|^{2}\left|(P \cdot y)^{\perp}\right|^{2}
$$

for all te $+y+w \in C M_{+}$. As a consequence, for $w \neq 0$,

$$
t=\frac{\sum_{i=0}^{m} w_{i} p_{i}}{2|w|^{2}}
$$

Moreover, the projection of $C M_{+}$onto the $T \oplus N$-space is the variety carved out by the equations

$$
|w|^{2}\left\langle P_{i} \cdot y, y\right\rangle-w_{i} \sum_{j=0}^{m} w_{j}\left\langle P_{j} \cdot y, y\right\rangle+2|w|^{2}\left\langle P_{i} \cdot y, w\right\rangle=0
$$

for all $i=0, \ldots, m$. 
Proof. The first two equations are apparently true for $w=0$. For $w \neq 0$, they follow from comparing the coefficients of (33) and (35), since both quadratic polynomials have the same double root. The third equation is a consequence of the first and (29). Finally, the last set of equations follow from (28) and the third equation.

Lemma 5. Notation as above, for $e \in M_{+}$, there is an open set $U$ in $T$ such that for each point $y \in U$, there are only a finite number of $w \in N$ (and hence finitely many $t$ ) for which te $+y+w \in M_{+}$with the property that these $w$ span $N$.

Proof. Consider the orthogonal projection $\pi: M_{+} \rightarrow T$ given by $t e+$ $y+w \mapsto y$. The map $\pi$ is surjective onto a neighborhood of $y=0$, because $\pi$ is in fact a local diffeomorphism near $e$ due to the fact that $T$ is tangent to $M_{+}$at $e$. By Sard's theorem, the regular values of $\pi$ in this neighborhood form a dense and open set $S$. Pick an open ball $U$ in $S$. The preimage of $\pi$ over each point in $U$ is finite with a fixed number of elements, so that $\pi$ is a covering map over $U$.

Suppose for some $y$ in $U$, the elements of $\pi^{-1}(y)$ is contained in a proper subplane $L$ of $\mathbb{R} e \oplus N$, then a slight perturbation from $y$ to a nearby $y^{\prime}$ in $T$ will disconnect the pertured image of the plane $\mathbb{R e} \oplus N$ from $M_{+}$, which contradicts the constancy of the number of elements of preimages near $y$, as a slight perturbation does not alter the intersection number. Therefore, the elements of $\pi^{-1}(y)$ span $\mathbb{R} e \oplus N$. However, since $t$ is a function of $y$ and $w \neq 0$ by (39), we see that the elements of $\pi^{-1}(y)$ projects to elements in $N$ which $\operatorname{span} N$.

Lemma 5 enables us to say more about (38) now.

Lemma 6. Let te $+y+w \in C M_{+}$. Then

$$
\sum_{i=0}^{m} q_{i} w_{i}=\sum_{i=0}^{m} p_{i}\left\langle P_{i} \cdot y, w\right\rangle,
$$

and

$$
|w|^{2}\left|(P \cdot y)^{\perp}\right|^{2}=\sum_{i=0}^{m}\left\langle P_{i} \cdot y, w\right\rangle^{2} .
$$


Proof. The identities are trivially true if $w=0$. We assume $w \neq 0$ now. Recall $P$ from (35). We claim that if $t \neq 0$, then

$$
\left|(P \cdot y)^{\perp}\right|^{2}=0
$$

if and only if

$$
\sum_{i=0}^{m}\left\langle P_{i} \cdot y, w\right\rangle^{2}=0
$$

To see this, note first of all, that $(P \cdot y)^{\perp}=0$ means $y$ belongs to the direct sum of the \pm 1 -eigenspace of the shape operator $A_{P \cdot e}$ at $e$. To see this, recall that $Q \cdot e$ traces out the unit normal sphere of $M_{+}$at $e$ as $Q$ varies in $\mathcal{C}$. Therefore, $(P \cdot y)^{\perp}=0$ gives

$$
0=\left\langle(P \cdot y)^{\perp}, Q \cdot e\right\rangle=\langle P \cdot y, Q \cdot e\rangle=\langle y, P Q \cdot e\rangle .
$$

However, since $P$ is a normal bundle isomorphism of $M_{+}, P$ maps the unit normal sphere at $e$ to that at $P \cdot e$. That is, $P Q \cdot e$ traces out the unit normal sphere at $P \cdot e$ as $Q$ varies in $\mathcal{C}$. On the other hand, the unit normal sphere at $P \cdot e$ generates $\mathbb{R} e \oplus E_{0}$, where $E_{0}$ is the 0 -eigenspace of $A_{P \cdot e}$, by Proposition 2. Hence, (45) asserts that $y$ belongs to the direct sum of the \pm 1 -eigenspace of the shape operator $A_{P \cdot e}$. In particular, (44) follows because it is equivalent to $\left\langle P_{i} \cdot y, P \cdot e\right\rangle=0, i=0, \ldots, m$, i.e.,

$$
\left\langle y, P_{i} P \cdot e\right\rangle=0
$$

for all $i$. However, we know $P_{i} P \cdot e$ are unit normal vectors at the point $P \cdot e$, by the construction of $\mathcal{C}$, which are thus vectors in $\mathbb{R} e \oplus E_{0}$. In particular, (46) and so (44) hold true if (43) does, proving one direction of the claim. Conversely, assume

$$
\sum_{i=0}^{m}\left\langle P_{i} \cdot y, w\right\rangle^{2}=0
$$

Set $r=:|w|, n=: w /|w|$ and $n_{i}=: w_{i} /|w|$. Substituting (39) into (33) and (35), with (37) in mind, we derive

(48) $0=4\left(\sum_{i=0}^{m}\left\langle P_{i} \cdot y, n\right\rangle^{2}\right) r^{2}-4\left(\sum_{i=0}^{m} p_{i}\left\langle P_{i} \cdot y, n\right\rangle\right) r-\langle P \cdot y, y\rangle^{2}+\sum_{0}^{m} p_{i}^{2}$, 
and

$$
0=4\left|(P \cdot y)^{\perp}\right|^{2} r^{2}-4\left(\sum_{i=0}^{m} q_{i} n_{i}\right) r-\langle P \cdot y, y\rangle^{2}+\sum_{0}^{m} p_{i}^{2},
$$

where we also employ the identity

$$
-\sum_{i=0}^{m} p_{i} w_{i}=r\langle P \cdot y, y\rangle
$$

which follows from (31).

We observe that (47) is equivalent to

$$
p_{i}(y)=2 t w_{i}
$$

for all $i$ by (28). Also,

$$
-(\langle P \cdot y, y\rangle)^{2}+\sum_{i=0}^{m} p_{i}^{2}=0
$$

because (47) implies the first two terms of (48) vanish, and so

$$
\left|(P \cdot y)^{\perp}\right|^{2}|w|^{2}=\sum_{i=0}^{m} q_{i} w_{i}
$$

hold by (49). Substituting (51) into the right hand side of (52), we obtain

$$
\left|(P \cdot y)^{\perp}\right|^{2}|w|^{2}=\frac{1}{2 t} \sum_{i=0}^{m} q_{i} p_{i}=0
$$

by the identity $\sum_{i=0}^{m} p_{i} q_{i}=0[3]$. Therefore,

$$
\left|(P \cdot y)^{\perp}\right|^{2}=0,
$$

and the claim is established.

By the claim, for $t \neq 0$, either both sides of (42) are zero, in which case (41) holds as well by (38), so that our proof is done, or we can from now on assume that both sides of (42) are nonzero. Now since

$$
\sum_{i=0}^{m}\left\langle P_{i} \cdot y, w\right\rangle^{2} \neq 0
$$


$\left\langle P_{i} \cdot y, w\right\rangle^{2} \neq 0$ for some $i$, for which (40), which is

$$
r^{2}\left\langle P_{i} \cdot y, y\right\rangle-r^{2} n_{i} \sum_{j=0}^{m} n_{j}\left\langle P_{j} \cdot y, y\right\rangle+2 r^{3}\left\langle P_{i} \cdot y, n\right\rangle=0,
$$

asserts that there is a single $r \neq 0$ satisfying both (48) and (49), so that in the case when

$$
-(\langle P \cdot y, y\rangle)^{2}+\sum_{i=0}^{m} p_{i}^{2} \neq 0,
$$

(48) and (49) have the same nonzero double roots. Hence, we compare the coefficients of (48) and (49) to conclude (41) and (42). On the other hand, if

$$
-(\langle P \cdot y, y\rangle)^{2}+\sum_{i=0}^{m} p_{i}^{2}=0
$$

then (50) implies

$$
\left(\sum_{i=0}^{m} p_{i} n_{i}\right)^{2}=\sum_{i=0}^{m} p_{i}^{2}
$$

from which the Cauchy-Schwarz inequality asserts that

$$
p_{i}=\lambda n_{i}
$$

for some $\lambda$, so that

$$
\left(\sum_{i=0}^{m} p_{i}\left\langle P_{i} \cdot y, n\right\rangle\right) r=\frac{\lambda}{r} \sum_{i=0}^{m} w_{i}\left\langle P_{i} \cdot y, w\right\rangle=0
$$

by (37). This forces $r=0$ by (53) and (48), which is absurd since $w \neq 0$. Hence, (41) and (42) are verified, when $t \neq 0$.

Lastly, we observe that the points in $C M_{+}$with $t=0$ is a proper subvariety in $C M_{+}$due to the nondegeneracy of $M_{+}$in the ambient sphere. Therefore, as the points $t e+y+w, t \neq 0$, approach points with $t=0$ in $C M_{+}$, we see by continuity that (41) and (42) remain true.

COROllary 5.

$$
\sum_{i=0}^{m} q_{i}(y) w_{i}=\left\langle\sum_{i=0}^{m} p_{i}(y) P_{i} \cdot y, w\right\rangle
$$

holds true for all $y \in T$ and all $w \in N$. 
Proof. By the preceding lemma, the same equation is valid for the finite $w$ over each $y$ in $U$ defined in Lemma 5. However, these finite $w$ generate the space $N$ for each $y \in U$ by Lemma 5 ; so the equality is true for all $N$ at each $y \in U$ since both sides of the equation are linear in $w$. Hence the equation must be true for all $T$ and $N$ since homogeneous polynomials are analytic.

Remark 3. In fact, one can see from [3] that

$$
F(y, y, y, w)=2 \sum_{i=0}^{m} q_{i}(y) w_{i}
$$

where $F\left(x_{1}, x_{2}, x_{3}, x_{4}\right)$ is the symmetric function associated with the Cartan-Münzner polynomial. Hence by (54), we have derived

$$
F(y, y, y, w)=2\left\langle\sum_{i=0}^{m} p_{i}(y) P_{i} \cdot y, w\right\rangle .
$$

\section{§6. The final argument}

Now we come to the crucial lemma.

Lemma 7. Let $\left(P_{0} \cdot e, \ldots, P_{m} \cdot e\right)$ and $\left(\bar{P}_{0} \cdot e, \ldots, \bar{P}_{m} \cdot e\right)$ be two orthonormal bases for the normal space $N$ to $M_{+}$at e, where $P_{0}, \ldots, P_{m}, \bar{P}_{0}, \ldots, \bar{P}_{m}$ $\in \mathcal{C}$. Let

$$
\bar{P}_{j} \cdot e=\sum_{i=0}^{m} A_{j}^{i}\left(P_{i} \cdot e\right)
$$

for some constant orthogonal matrix $\left(A_{j}^{i}\right)$. Then

$$
\bar{P}_{j}=\sum_{i=0}^{m} A_{j}^{i} P_{i}
$$

Proof. By (55), we have

$$
F(y, y, y, w)=\left\langle\sum_{i=0}^{m} p_{i}(y) P_{i} \cdot y, w\right\rangle=\left\langle\sum_{i=0}^{m} \bar{p}_{i}(y) \bar{P}_{i} \cdot y, w\right\rangle .
$$

Now since

$$
p_{i}(y)=\left\langle S(y, y), P_{i} \cdot e\right\rangle
$$


by (30) and (32), we have immediately

$$
\bar{p}_{j}(y)=\sum_{i=0}^{m} A_{j}^{i} p_{i}(y)
$$

which results in, by (57),

$$
\left\langle\sum_{i=0}^{m} p_{i}(y)\left(P_{i}-\sum_{j=0}^{m} A_{j}^{i} \bar{P}_{j}\right) \cdot y, w\right\rangle=0
$$

for all $y \in T$ and $w \in N$. For ease of notation, set

$$
M_{i}=: P_{i}-\sum_{j=0}^{m} A_{j}^{i} \bar{P}_{j} .
$$

In particular, (58) implies that

$$
\left(\sum_{i=0}^{m} p_{i}(y) M_{i}\right) \cdot y \in T
$$

Hence

$$
\left(\sum_{i=0}^{m} p_{i}(y) M_{i}\right) \cdot y=\left(\sum_{i=0}^{m} p_{i}(y) M_{i}^{T}\right) \cdot y=0,
$$

where the superscript $T$ denotes the orthogonal projection onto $T$. That it is equal to zero comes from the fact that, e.g., $P_{i}^{T}$ is just the shape operator $A_{P_{i} \cdot e}$, and therefore the correct transformation compatible with (56) prevails. We conclude that

$$
\left(\sum_{i=0}^{m} p_{i}(y) M_{i}\right) \cdot y=0
$$

for all $y \in T$. On the other hand,

$$
\left(\sum_{i=0}^{m} p_{i}(y) M_{i}\right) \cdot w=0
$$

for all $w \in N$. This is because first of all, e.g., $\left\langle P_{i} \cdot w, P_{j} \cdot e\right\rangle=0$. For, again $P_{i} P_{j} \cdot e$ is in the span of $e$ and the 0 -eigenspace of $A_{P_{j} \cdot e}$, so that as a result $\left(\sum_{i=0}^{m} p_{i}(y) M_{i}\right) \cdot w$ is perpendicular to the normal space $N$; moreover,

$$
\left\langle\left(\sum_{i=0}^{m} p_{i}(y) M_{i}\right) \cdot w, y\right\rangle=\left\langle\left(\sum_{i=0}^{m} p_{i}(y) M_{i}\right) \cdot y, w\right\rangle=0
$$


by (59) and the fact that the operators involved are symmetric, so that $\left(\sum_{i=0}^{m} p_{i}(y) M_{i}\right) \cdot w$ is also perpendicular to $T$. Lastly

$$
\left\langle\left(\sum_{i=0}^{m} p_{i}(y) M_{i}\right) \cdot w, e\right\rangle=0
$$

since

$$
\left(\sum_{i=0}^{m} p_{i}(y) M_{i}\right) \cdot e=0
$$

automatically by (56). The upshot is that

$$
\sum_{i=0}^{m} p_{i}(y) M_{i}=0
$$

for all $y \in T$.

We are now in the situation where we have $m+1$ constant matrices $M_{0}, \ldots, M_{m}$ such that

$$
\sum_{i=0}^{m} p_{i}(y) M_{i}=0
$$

for all $y \in T$. If one of $M_{i}$ is nonzero, we will find constants $c_{0}, \ldots, c_{m}$, not all zero, such that

$$
\sum_{i=0}^{m} c_{i} p_{i}(y)=0
$$

for all $y \in T$, by looking at an appropriate matrix entry. In other words, the symmetric matrix

$$
M=: \sum_{i=0}^{m} c_{i} A_{P_{i} \cdot e}
$$

( $A$ is the shape operator) satisfies $\langle M \cdot y, y\rangle=0$ for all $y$. Thus $M=0$, which implies that the shape operator $A_{n}$, where $n$ is the unit normal vector normalized from the vector $\left(\sum_{i=0}^{m} c_{i} P_{i}\right) \cdot e$, will be identically zero. This is a contradiction, since we know all the shape operators for $M_{+}$have $0, \pm 1$ as eigenvalues. In conclusion, all $M_{i}=0$, which is what we want to prove.

THEOREM 1. $\mathcal{C}$ is the Clifford sphere if and only if (17) through (20) hold. 
Proof. This follows immediately from the preceding lemma, because it says that $\mathcal{C}$ is the round sphere in the space of symmetric endomorphisms, if (17) through (20) hold. Conversely, we have seen that an isoparametric hypersurface of FKM-type satisfies (17) through (20).

\section{REFERENCES}

[1] T. Cecil, Lie Sphere Geometry, Springer-Verlag, New York Inc., 1992.

[2] T. Cecil, Q.-S. Chi and G. Jensen, Isoparametric hypersurfaces with four principal curvatures, Ann. Math., 166 (2007), 1-76.

[3] H. Ozeki and M. Takeuchi, On some types of isoparametric hypersurfaces in spheres I, Tohôku Math. J., 27 (1975), 515-559; II, Tohôku Math. J., 28 (1976), 7-55.

[4] D. Ferus, H. Karcher and H.-F. Münzner, Cliffordalgebren und neue isoparametrische Hyperflächen, Math. Z., 177 (1981), 479-502.

[5] R. Miyaoka, Dupin hypersurfaces and a Lie invariant, Kodai Math. J., 12 (1989), $228-256$.

[6] M. Spivak, A Comprehensive Introduction to Differential Geometry, Vol. IV, 2nd ed., Publish or Perish Inc., Berkeley, 1979.

Department of Mathematics

Washington University

St. Louis, MO 63130

U.S.A.

chi@math. wustl.edu 